\title{
Spin Dynamics of Double-Exchange Manganites with Magnetic Frustration
}

\author{
R.S. Fishman \\ Condensed Matter Sciences Division, \\ Oak Ridge National Laboratory, Oak Ridge, TN 37831-6032
}

\begin{abstract}
This work examines the effects of magnetic frustration due to competing ferromagnetic and antiferromagnetic Heisenberg interactions on the spin dynamics of the double-exchange model. When the local moments are non-colinear, a charge-density wave forms because the electrons prefer to sit on lines of sites that are coupled ferromagnetically. With increasing hopping energy, the local spins become aligned and the average spin-wave stiffness increases. Phase separation is found only within a narrow range of hopping energies. Results of this work are applied to the field-induced jump in the spin-wave stiffness observed in the manganite $\operatorname{Pr}_{1-x} \mathrm{Ca}_{x} \mathrm{MnO}_{3}$ with $0.3 \leq x \leq 0.4$.
\end{abstract}


The persistence of antiferromagnetic (AFM) short-range order below the Curie temperarture $T_{\mathrm{C}}$ of the manganites has been known for many years 1 ]. Close to but below $T_{\mathrm{C}}$, metallic manganites like $\mathrm{La}_{0.7} \mathrm{Ca}_{0.3} \mathrm{MnO}_{3}$ contain polaronic regions [2, 3] that are responsible for the coexistence of propagating and diffusive spin dynamics [4]. In the remarkable compound $\operatorname{Pr}_{1-x} \mathrm{Ca}_{x} \mathrm{MnO}_{3}$ with $0.3 \leq x \leq 0.4$, the low-temperature ferromagnetic (FM) insulating phase was originally believed to be a canted AFM (CAF) [5, 6] but probably contains regions with both FM and AFM short-range order [7, 8]. When an applied field $B$ exceeds about $3 \mathrm{~T}$, the resistivity drops by several orders of magnitude [5], the AFM regions shrink [8], and the spin-wave (SW) stiffness $D_{\mathrm{sw}}$ jumps by a factor of 3 [9]. Despite the recognition that short-range AFM order plays a central role in the manganites, little is known theoretically about how long-wavelength SW's are affected by propagating through both FM and AFM regions. Because electron hopping is hampered by the misalignmnent of the local moments 10], AFM interactions may be expected to suppress the contribution of electron-mediated double-exchange (DE) to the SW dynamics [11]. This paper examines the effects of AFM interactions and non-colinearity on the SW dynamics of electrons coupled to the local moments of a generalized Villain model $12,13,14]$. Our results strongly suggest that the jump in the SW stiffness observed [9] in $\mathrm{Pr}_{0.7} \mathrm{Ca}_{0.3} \mathrm{MnO}_{3}$ is produced by a sharp increase in the hopping energy at the critical field rather than by the alignment of the AFM regions.

As pictured in Fig.1(a), the local moments $\mathbf{S}_{i}$ of the generalized Villain model are coupled by the FM interaction $J$ along the $\hat{\mathbf{y}}$ direction and by either the FM interaction $J$ or the AFM interaction $-\eta J$ along the $\hat{\mathbf{x}}$ direction. A zero-temperature CAF phase is stabilized when $\eta$ exceeds $\eta_{c}$, which is $1 / 3$ when $\mathbf{B}=B \hat{\mathbf{z}}=0$ but increases as $B$ increases. Villain's original model [12] set $\eta=1$, which is the condition for full frustration. Due to the different environments of the $a$ and $b$ sites, the angle $\theta_{b}$ at the $b$ sites is always larger than $\theta_{a}$ at the $a$ sites, as shown in Fig.1(b).

Within our hybrid model, the Heisenberg interactions between the local moments are given by the generalized Villain model while electrons with density $p=1-x$ are FM coupled to the local moments by Hund's coupling $J_{\mathrm{H}}$ and hop between neighboring sites with energy $t$. The DEV model (so called because it combines the DE and generalized Villain models) provides several advantages as a basis for understanding the effects of magnetic frustration on the spin dynamics. First, it is one of the simplest periodic models that is 
(a)

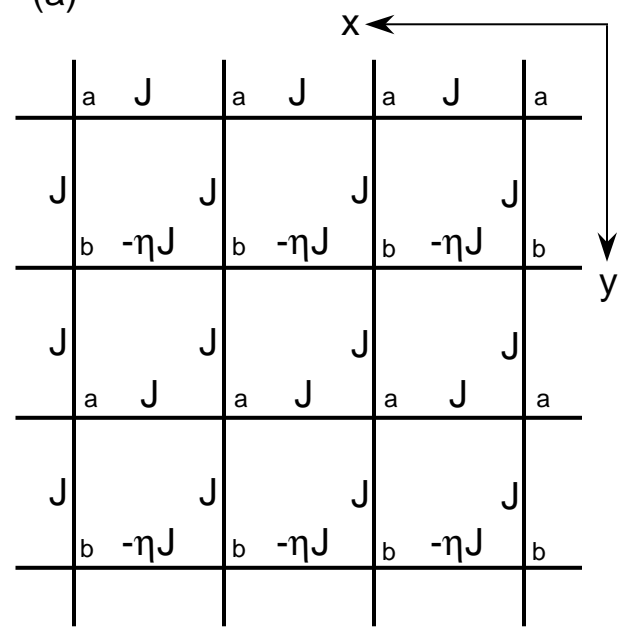

(b)

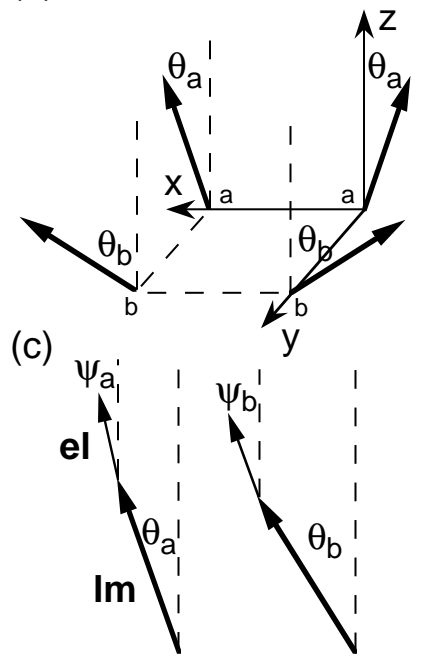

FIG. 1: (a) The generalized Villain model with Heisenberg couplings $J$ or $-\eta J$, (b) the local moments in the $x z$ plane subtend angles $\theta_{a}$ and $\theta_{b}$ with the $z$ axis, and (c) the electron spins also lie in the $x z$ plane but subtend angles $\psi_{a}<\theta_{a}$ and $\psi_{b}<\theta_{b}$ with the $z$ axis.

magnetically frustrated, which can be controlled through the parameter $\eta$. In contrast to the case in a DE model with AFM interactions between all neighboring local moments [15, 16], a homogeneous CAF phase is stable against phase separation [17] except in a very narrow region of parameter space. Second, unlike a model with AFM exchange only, the DEV model supports FM order even when $t=0$ and $B=0$. So it can be used to track the change in SW stiffness $D_{\mathrm{sw}}$ as the electrons become mobile. Third, because it contains both FM and AFM Heisenberg interactions, the DEV model can be used to study insulating manganites like $\operatorname{Pr}_{0.66} \mathrm{Ca}_{0.34} \mathrm{MnO}_{3}$, where the AFM interactions arise from superexchange and the FM interactions from short-range orbital and polaronic order [18, 19].

For simplicity, our model is translationally symmetric with the FM and AFM Heisenberg couplings arranged periodically in two dimensions. In the low-temperature phase of $\operatorname{Pr}_{0.67} \mathrm{Ca}_{0.33} \mathrm{MnO}_{3}$, the $\mathrm{FM}$ interactions may be confined to two-dimensional sheets in a "red cabbage" structure [8]. But for wavelengths longer than the thickness $\sim 25 \AA$ of the FM sheets, the SW's will average over the FM and AFM regions. So the DEV model will provide qualitiatively accurate predictions for the average SW stiffness $D_{\mathrm{sw}}^{a v}=\left(D_{\mathrm{sw}}^{x}+D_{\mathrm{sw}}^{y}\right) / 2$, which is defined in the long-wavelength limit. 
The Hamiltonian of the DEV model is

$$
H=-t \sum_{\langle i, j\rangle} \sum_{\alpha}\left(c_{i \alpha}^{\dagger} c_{j \alpha}+c_{j \alpha}^{\dagger} c_{i \alpha}\right)-2 J_{\mathrm{H}} \sum_{i} \mathbf{s}_{i} \cdot \mathbf{S}_{i}-\sum_{\langle i, j\rangle} J_{i j} \mathbf{S}_{i} \cdot \mathbf{S}_{j}-B \sum_{i}\left(S_{i z}+s_{i z}\right)
$$

where $c_{i \alpha}^{\dagger}$ and $c_{i \alpha}$ are the creation and destruction operators for an electron with spin $\alpha$ at site $i, \mathbf{s}_{i}=(1 / 2) c_{i \alpha}^{\dagger} \sigma_{\alpha \beta} c_{i \beta}$ is the electronic spin, and $\mathbf{S}_{i}$ is the spin of the local moment with magnitude $S$. The Heisenberg interactions $J_{i j}$ take the values $J$ or $-\eta J$, as described in Fig.1(a). This model will be solved at zero temperature to lowest order in $1 / S$. To guarantee that the contributions to the SW frequencies from DE hopping $t$ and from the Heisenberg interactions $J_{i j}$ are of the same order in $1 / S, t$ is considered to be of the same order in $1 / S$ as $J_{\mathrm{H}} S, J S^{2}$ and $B S$ (although their relative values can be quite different). Thus, the dimensionless parameters of the DEV model are $t^{\prime}=t / J S^{2}, \eta, B^{\prime}=B / J S$, and $J_{\mathrm{H}} / J S$. To lowest order in $1 / S$, the magnetic field $B$ only couples to the local moments. While the theory developed below can be extended to treat all values of the Hund's coupling, for simplicity we shall consider the limit of large $J_{\mathrm{H}} S$ or in dimensionless terms, $J_{\mathrm{H}} / J S \gg 1$ and $J_{\mathrm{H}} S / t \gg 1$.

To solve this model, a Holstein-Primakoff expansion is first performed within the rotated reference frame of each spin: $\bar{S}_{i z}=S-a_{i}^{\dagger} a_{i}, \bar{S}_{i+}=\sqrt{2 S} a_{i}$, and $\bar{S}_{i-}=\sqrt{2 S} a_{i}^{\dagger}$. In terms of electronic creation and destruction operators $\bar{c}_{\mathbf{k}, \alpha}^{(r) \dagger}$ and $\bar{c}_{\mathbf{k}, \alpha}^{(r)}$ in the rotated reference frame of the local moments, the zeroth-order term (in powers of $1 / \sqrt{S}$ ) in the Hamiltonian can be written as $H_{0}=E_{h}+H_{b}$ where to lowest order in $t / J_{\mathrm{H}} S$,

$$
\begin{gathered}
E_{h}=\frac{1}{2} N J S^{2}\left\{-\cos 2 \theta_{a}+\eta \cos 2 \theta_{b}-2 \cos \left(\theta_{a}-\theta_{b}\right)-B^{\prime}\left(\cos \theta_{a}+\cos \theta_{b}\right)\right\}, \\
H_{b}=\sum_{\mathbf{k}, \alpha}\left\{\bar{c}_{\mathbf{k} \alpha}^{(a) \dagger} \bar{c}_{\mathbf{k} \alpha}^{(a)}\left(-J_{\mathrm{H}} S \alpha-2 t \cos k_{x} \cos \theta_{a}\right)+\bar{c}_{\mathbf{k} \alpha}^{(b) \dagger} \bar{c}_{\mathbf{k} \alpha}^{(b)}\left(-J_{\mathrm{H}} S \alpha-2 t \cos k_{x} \cos \theta_{b}\right)\right. \\
\left.-\left(\bar{c}_{\mathbf{k} \alpha}^{(a) \dagger} \bar{c}_{\mathbf{k} \alpha}^{(b)}+\bar{c}_{\mathbf{k} \alpha}^{(b) \dagger} \bar{c}_{\mathbf{k} \alpha}^{(a)}\right) 2 t \cos k_{y} \cos \left(\left(\theta_{a}-\theta_{b}\right) / 2\right)\right\} .
\end{gathered}
$$

Here, the lattice constant is set to 1 and $\alpha= \pm 1$ corresponds to spin up or down in the local reference frames.

The electronic Hamiltonian $H_{b}$ is easily transformed into the diagonal form $H_{b}=$ $\sum_{\mathbf{k}, \alpha, r} \epsilon_{\mathbf{k} \alpha}^{(r)} d_{\mathbf{k} \alpha}^{(r) \dagger} d_{\mathbf{k} \alpha}^{(r)}$ by the rotations $\bar{c}_{\mathbf{k} \alpha}^{(a)}=u_{\mathbf{k}}^{(a)} d_{\mathbf{k} \alpha}^{(a)}+u_{\mathbf{k}}^{(b)} d_{\mathbf{k} \alpha}^{(b)}$ and $\bar{c}_{\mathbf{k} \alpha}^{(b)}=u_{\mathbf{k}}^{(b)} d_{\mathbf{k} \alpha}^{(a)}-u_{\mathbf{k}}^{(a)} d_{\mathbf{k} \alpha}^{(b)}$ where $\epsilon_{\mathbf{k} \alpha}^{(r)}=-J_{\mathrm{H}} S \alpha+\tilde{\epsilon}_{\mathbf{k}}^{(r)}, u_{\mathbf{k}}^{(a) 2}=1-u_{\mathbf{k}}^{(b) 2}=\left(1+\left(\cos \theta_{a}-\cos \theta_{b}\right) \cos k_{x} / w_{\mathbf{k}}\right) / 2$,

$$
\tilde{\epsilon}_{\mathbf{k}}^{(r)}=-t \cos k_{x}\left(\cos \theta_{a}+\cos \theta_{b}\right) \mp t w_{\mathbf{k}},
$$




$$
w_{\mathbf{k}}=\sqrt{\left(\cos \theta_{a}-\cos \theta_{b}\right)^{2} \cos ^{2} k_{x}+4 \cos ^{2}\left(\left(\theta_{a}-\theta_{b}\right) / 2\right) \cos ^{2} k_{y}} .
$$

The $\mp$ signs refer to the $r=a$ and $b$ bands, respectively, and the first Brillouin zone extends from $-\pi$ to $\pi$ in the $k_{x}$ direction but only from $-\pi / 2$ to $\pi / 2$ in the $k_{y}$ direction due to the reduced symmetry.

In the limit of large $J_{\mathrm{H}} S$, the zeroth-order energy $E_{0}=E_{h}+\left\langle H_{b}\right\rangle$ can readily be minimized with respect to the angles $\theta_{a}$ and $\theta_{b}$. When $t=B=0, \theta_{b}=3 \theta_{a}$ for all $\eta$. For a fixed $\eta$ and $B^{\prime}$, the equilibrium angles decrease with increasing $t^{\prime}$ and $\theta_{b}<3 \theta_{a}$. The phase boundary between the CAF and FM phases satisfies the condition

$$
B^{\prime}-2 \eta+4+3 E_{\mathrm{ke}} / 4 J S^{2}-2 \sqrt{(1+\eta)^{2}+\left(1+E_{\mathrm{ke}} / 8 J S^{2}\right)^{2}}=0
$$

where $E_{\mathrm{ke}}=-\left(\left\langle\tilde{\epsilon}_{\mathrm{k}}^{(a)}\right\rangle+\left\langle\tilde{\epsilon}_{\mathrm{k}}^{(b)}\right\rangle\right) / 2>0$ is the average kinetic energy of the electrons in the FM phase. For $p=0.66, \eta=3$, and $B=0$, the dependence of the equilibrium angles on $t^{\prime}$ is plotted in Fig.2(a). Also shown is the average spin $M=S\left(\cos \theta_{a}+\cos \theta_{b}\right) / 2$ of the local moments.

Surprisingly, the electronic occupation of the $a$ and $b$ sites are different with most of the electrons sitting on the $a$ sites. The fraction $f_{a}$ of such electrons, also plotted in Fig.2(a), has a maximum of 0.58 as $t^{\prime} \rightarrow 0$ and approaches $1 / 2$ as $t^{\prime} \rightarrow t_{c}^{\prime} \approx 21.2$. This behavior is easy to understand: the largest angles between neighboring spins are along the $x$ axis between $b$ sites with angles differing by $2 \theta_{b}$. When an electron hops onto a $b$ site, it cannot easily hop to other $b$ sites and so quickly moves onto a neighboring $a$ site, where it can readily travel between other $a$ sites with angular difference $2 \theta_{a} \ll 2 \theta_{b}$. Hence, the non-colinearity of the local moments quite naturally produces a charge-density wave (CDW) with a substantial amplitude. A CDW with the same period as the one predicted here has in fact been observed in the insulating phase of $\mathrm{Pr}_{0.7} \mathrm{Ca}_{0.3} \mathrm{MnO}_{3}[6,20]$.

Another surprise is that phase separation occurs within a narrow range of $t^{\prime}$ around 10.0. Phase separation is easily seen in a plot of filling $p$ versus chemical potential $\mu$ as a discontinuity $\Delta p$ in $p(\mu)$. It appears at fixed $p$ as jumps in the equilibrium angles $\theta_{r}$ and electron fraction $f_{a}$. Like the Pomeranchuk instability [21] in the two-dimensional Hubbard model, the phase instability in the DEV model occurs close to a Van Hove filling and is marked by a change in Fermi surface (FS) topology from closed to open, as shown in the inset to Fig. 3 where the FS is sketched for values of $t^{\prime}$ on either side of the phase-separated range. For $t^{\prime}=10.2$, the extra electrons in the neck of the $a$ FS around $\mathbf{k}=0$ are cancelled 

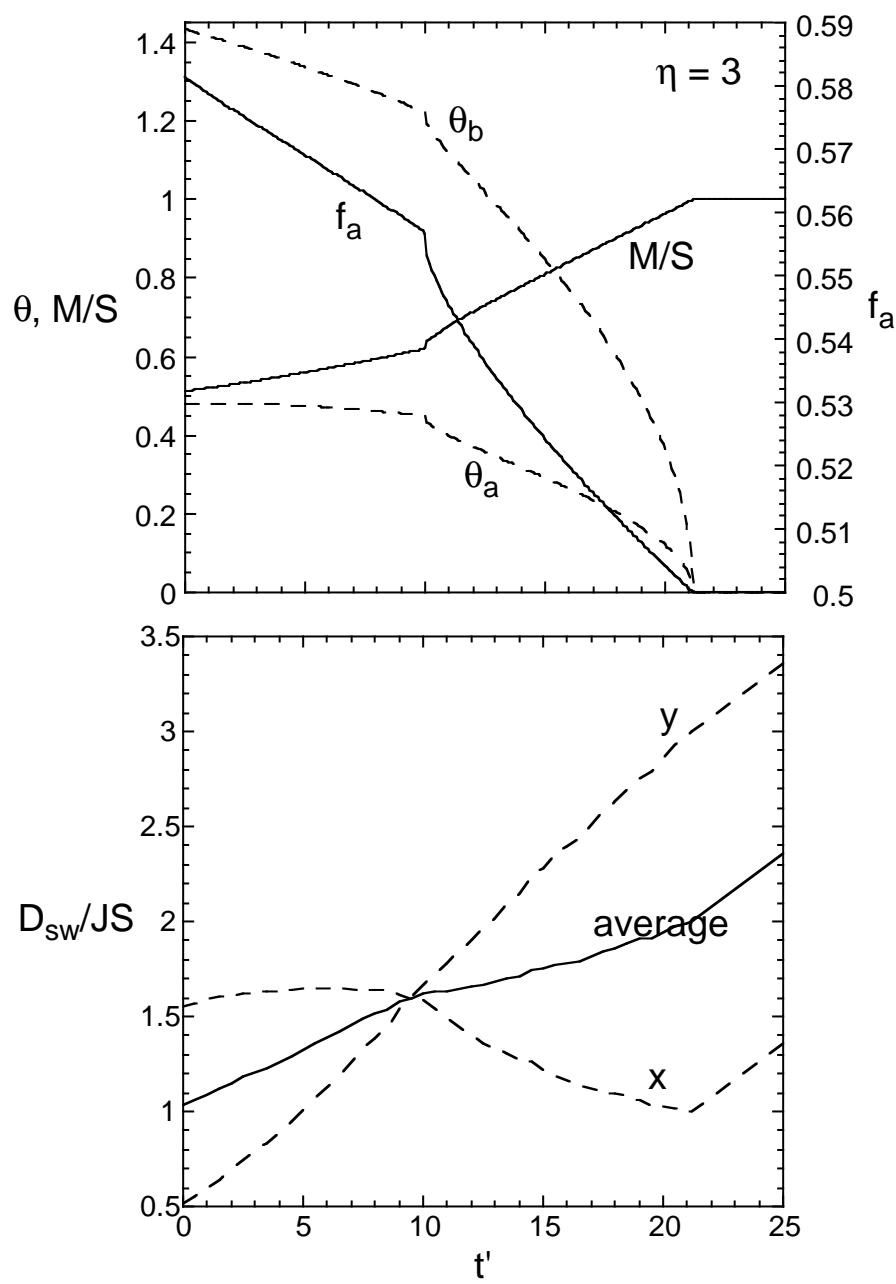

FIG. 2: (a) The angles $\theta_{a}$ and $\theta_{b}$ of the local moments, the total local magnetization $M / S$, the fraction $f_{a}$ of the electrons on the $a$ sites, and (b) the SW stiffnesses for $p=0.66, \eta=3$ and $B=0$ versus $t^{\prime}$.

by the holes in the $b \mathrm{FS}$ around $\mathbf{k}=(\pi, \pi / 2)$. However, the phase separation is extremely weak and for the parameters in Fig. $2, \Delta p \approx 0.003$.

As sketched in Fig.1(c), the equilibrium angles $\psi_{r}$ for the electrons are not equal to the angles $\theta_{r}$ of the local moments except when $J_{\mathrm{H}} S / t=\infty$. For finite $J_{\mathrm{H}} S / t, \psi_{r}<\theta_{r}$ as the electrons try to align their spins as much as possible. In the limit of large Hund's coupling, $\theta_{r}-\psi_{r} \propto t / J_{\mathrm{H}} S$ and the electrons always exert a small torque on the local moments. Hence, the relationships given above for $\bar{c}_{\mathbf{k} \alpha}^{(r)}$ in terms of $d_{\mathbf{k} \alpha}^{(s)}$ should contain admixtures of opposite-spin terms like $\left(t / J_{\mathrm{H}} S\right) d_{\mathbf{k}+\mathbf{Q},-\alpha}^{(s)}$, where $\mathbf{Q}=(\pi, 0)$ is the AFM Bragg vector. Since 


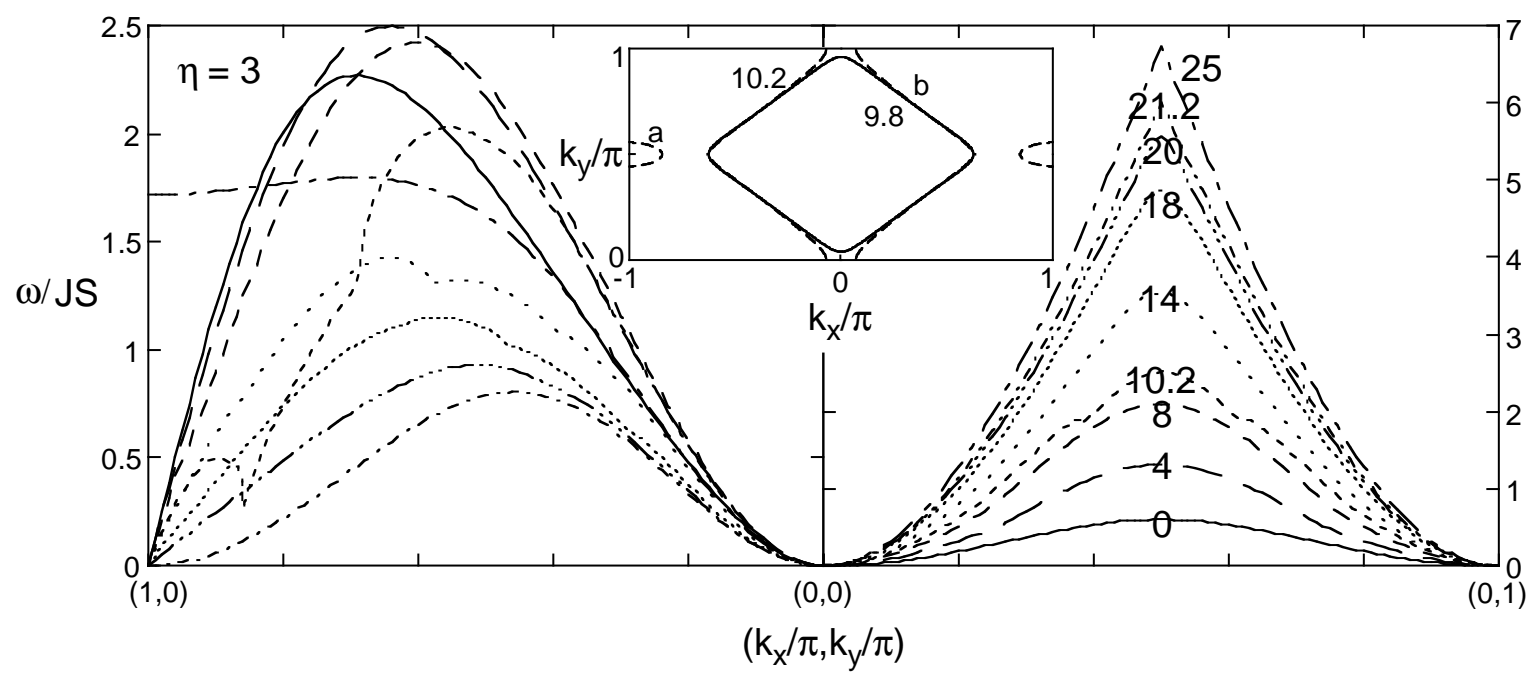

FIG. 3: The SW frequencies for $p=0.66, \eta=3, B=0$, and various values of $t^{\prime}$. Different energy scales are used on either side of $\mathbf{k}=0$. In the inset, we plot the FS for $t^{\prime}=9.8$ (solid) and 10.2 (dashed), on either side of a narrow region of phase separation.

$\left\langle\bar{s}_{i x}\right\rangle \propto \sin \left(\theta_{i}-\psi_{i}\right)$, these new terms produce a correction to the Hund's coupling $-2 J_{\mathrm{H}} \mathbf{S}_{i} \cdot \mathbf{s}_{i}$ that survives in the $J_{\mathrm{H}} S \rightarrow \infty$ limit.

After diagonalizing the band Hamiltonian $H_{b}$, the full Hamiltonian can be expanded as a power series in $1 / \sqrt{S}$ : $H=H_{0}+H_{1}+H_{2}+\cdots$, where $H_{1}$ contains the torque terms and is linear in the boson operators $a_{\mathbf{k}}^{(r)}$. To eliminate this first-order term and to express the Hamiltonian in terms of the true SW operators for the total spin $\mathbf{S}_{i \text {,tot }}=\mathbf{S}_{i}+\mathbf{s}_{i}$, we perform the unitary transformation [16] $H^{\prime}=e^{-U} H e^{U}$ where $U$ is constructed to satisfy $\left[U, H_{0}\right]=H_{1}$. To lowest order in $1 / S$, a trace over the Fermion degrees of freedom yields the modified second-order Hamiltonian $H_{2}^{\prime}=H_{2}+\left[U, H_{1}\right] / 2$ for the SW operators only:

$$
H_{2}^{\prime}=J S \sum_{\mathbf{k}, r, s}\left\{a_{\mathbf{k}}^{(r) \dagger} a_{\mathbf{k}}^{(s)} A_{\mathbf{k}}^{(r, s)}+\left(a_{-\mathbf{k}}^{(r)} a_{\mathbf{k}}^{(s)}+a_{-\mathbf{k}}^{(r) \dagger} a_{\mathbf{k}}^{(s) \dagger}\right) B_{\mathbf{k}}^{(r, s)}\right\}
$$

It is then straightforward to diagonalize $H_{2}^{\prime}$ to obtain the mode frequencies $\omega_{\mathbf{k}}$. The torque terms discussed above are required to preserve rotational symmetry and the relations $\omega_{\mathbf{k}=0}=$ $B$ and $\omega_{\mathbf{Q}}=0$ in the CAF phase.

In the FM phase, the SW frequency is given by the analytic result

$$
\begin{aligned}
\omega_{\mathbf{k}}= & B+J S\left(3-\eta+(\eta-1) \cos k_{x}\right)+E_{\mathrm{ke}}\left(2-\cos k_{x}\right) / 4 S \\
& -J S \sqrt{(1+\eta)^{2}\left(1-\cos k_{x}\right)^{2}+4\left(1+E_{\mathrm{ke}} / 8 J S^{2}\right)^{2} \cos ^{2} k_{y} .}
\end{aligned}
$$


An AFM component develops when $\omega_{\mathbf{Q}}=0$, which yields the same condition for the phase boundary as Eq.(6). For the FM phase, the SW stiffness obtained from the long-wavelength expansion $\omega_{\mathrm{k}} \approx B+D_{\mathrm{sw}}^{x} k_{x}^{2}+D_{\mathrm{sw}}^{y} k_{y}^{2}$ is simply the sum of the DE and Heisenberg contributions: $D_{\mathrm{sw}}^{x}=E_{\mathrm{ke}} / 8 S+J S(1-\eta) / 2$ and $D_{\mathrm{sw}}^{y}=E_{\mathrm{ke}} / 8 S+J S$. For $J=0$, these results agree with the SW frequencies of the DE model first obtained by Furukawa [22].

In the CAF phase, the SW frequency and stiffness must be solved numerically. When $t=0$, our results agree with Saslow and Erwin [14] for the generalized Villain model. Results for $\omega_{\mathbf{k}}$ are plotted in Fig.3 for $p=0.66, \eta=3, B^{\prime}=0$, and various values of $t^{\prime}$. Above the phase separation region around $t^{\prime} \approx 10.0$ but below $t_{c}^{\prime} \approx 21.2, \omega_{\mathrm{k}}$ develops kinks that correspond to transitions across the neck of the $a \mathrm{FS}\left(\mathbf{Q}-\mathbf{k} \approx 0.14 \pi \hat{\mathbf{x}}\right.$ for $\left.t^{\prime}=10.2\right)$ and the length of the $b$ FS $\left(\mathbf{Q}-\mathbf{k} \approx 0.31 \pi \hat{\mathbf{x}}\right.$ for $\left.t^{\prime}=10.2\right)$.

As plotted in Fig.2(b), the SW stiffness in the $\hat{\mathbf{x}}$ direction reaches a minimum at $t_{c}^{\prime}$, above which both $D_{\mathrm{sw}}^{x}$ and $D_{\mathrm{sw}}^{y}$ are linearly increasing functions of $t^{\prime}$. The stiffnesses in the $\hat{\mathbf{x}}$ and $\hat{\mathbf{y}}$ directions cross in the region of phase separation, where the SW's are isotropic in the long-wavelength limit. Notice that $D_{\mathrm{sw}}^{a v}$ increases by roughly a factor of 2 as $t^{\prime}$ increases from zero to $t_{c}^{\prime}$ and the system transforms from a CAF with a CDW into a FM.

By contrast, the effect of a magnetic field is quite different. After a sudden increase of the SW stiffness for very small fields that occurs in any CAF [23], there is a gradual increase in $D_{\mathrm{sw}}^{y}$ and decrease in $D_{\mathrm{sw}}^{x}$ as $B$ increases to $B_{c}$. For $t^{\prime}=3$ and $\eta=2, D_{\mathrm{sw}}^{a v}$ drops from $1.2 J S$ at $B=0$ to $0.54 J S$ at $B_{c}$. A field also very quickly eliminates the region of phase separation.

These results clearly indicate that the jump in SW stiffness observed [9] in $\mathrm{Pr}_{0.7} \mathrm{Ca}_{0.3} \mathrm{MnO}_{3}$ at a field of $3 \mathrm{~T}$ cannot be produced by simply aligning the AFM regions while keeping the bandwidth $\sim t$ fixed. The identical resistivities in the metal-insulator transition produced by either a magnetic field or x-rays 24] suggest a common mechanism: the excitation of charge carriers out of polaronic traps formed by the electron-lattice coupling. The doubling of $D_{\mathrm{sw}}^{a v}$ found in Fig.2(b) provides strong support for this scenario. Since the integrated optical weight is proportional to $E_{\mathrm{ke}} \sim t$, the jump in the hopping energy at $3 \mathrm{~T}$ should be reflected in the optical conductivity. Measurements by Okimoto et al. [6] on $\mathrm{Pr}_{0.6} \mathrm{Ca}_{0.4} \mathrm{MnO}_{3}$ do reveal a large increase in $\sigma(\omega)$ and a rapid drop in the CDW gaps near the critical field. If the percolation threshold for the FM regions [25] is exceeded when the electrons delocalize, then the jump in the SW stiffness will coincide with the metal-insulator transition. Otherwise 
the metal-insulator transition will occur at a slightly higher field.

Because it requires two sublattices with filling $x=0.5$, local CE-type AFM ordering in the manganites $\mathrm{Pr}_{0.6} \mathrm{Ca}_{0.4} \mathrm{MnO}_{3}$ [6, 20] and $\mathrm{La}_{0.7} \mathrm{Ca}_{0.3} \mathrm{MnO}_{3}$ [2, 3] would be simplified if the polaronic regions were rich in holes and poor in electrons. The DEV model provides a natural explanation for this behavior, since the electronic fraction on $b$ sites is substantially smaller than the fraction on $a$ sites as the electrons avoid regions with more pronounced AFM order.

To conclude, we have studied the effect of AFM interactions on the magnetic order and SW dynamics of electrons interacting with the local moments of a generalized Villain model. This model contains rich physics that provides insight into the SW dynamics of any itinerant system with competing FM and AFM Heisenberg interactions.

This research was sponsored by the U.S. Department of Energy under contract DEAC05-00OR22725 with Oak Ridge National Laboratory, managed by UT-Battelle, LLC. Conversations with Drs. A. Chernyshev, J. Fernandez-Baca, M. Katsnelson, N. Furukawa, W. Saslow, R. Wood, and A. Zheludev are happily acknowledged.

[1] For an overview of the manganites and issues of phase separation, see E. Dagotto, T. Hotta, and A. Moreo, Phys. Rep. 233, 1 (2001).

[2] C.P. Adams, J.W. Lynn, Y.M. Mukovskii, A.A. Arsenov, and D. Shulyatev, Phys. Rev. Lett. 85, 3954 (2000).

[3] T.Y. Koo, V. Kiryukhin, P.A. Sharma, J.P. Hill, and S.-W. Cheong, Phys. Rev. B 64, 220405 (2001).

[4] A. Chernyshev and R.S. Fishman, Phys. Rev. Lett. 90, 177202 (2003).

[5] H. Yoshizawa, H. Kawano, Y. Tomioka, and Y. Tokura, Phys. Rev. B 52, R13145 (1995); J. Phys. Soc. Japan 65, 1043 (1996).

[6] Y. Okimoto, Y. Tomioka, Y. Onose, Y. Otsuka, and Y. Tokura, Phys. Rev. B 59, 7401 (1999).

[7] I.G. Deac, J.F. Mitchell, and P. Schiffer, Phys. Rev. B 63, 172408 (2001).

[8] Ch. Simon, S. Mercone, N. Guiblin, C. Martin, A. Brûlet, and G. André, Phys. Rev. Lett. 89, $207202(2002)$.

[9] J.A. Fernandez-Baca, P.-C. Dai, H. Kawano-Furukawa, H. Yoshizawa, E.W. Plummer, S. 
Katano, Y. Tomioka, and Y. Tokura, Phys. Rev. B 66, 054434 (2002).

[10] As found by P.W. Anderson and H. Hasegawa, Phys. Rev. 100, 675 (1955), the effective hopping of electrons between two local moments making an angle $\Theta$ is reduced from $t$ to $t \cos (\Theta / 2)$ in the limit of large $J_{\mathrm{H}} S$.

[11] P.-G. DeGennes, Phys. Rev. 118, 141 (1960).

[12] J. Villain, J. Phys. C 10, 1717 (1977).

[13] M. Gabay, T. Garel, G.N. Parker, and W.M. Saslow, Phys. Rev. B 40, 264 (1989).

[14] W.M. Saslow and R. Erwin, Phys. Rev. B 45, 4759 (1992).

[15] M.Yu. Kagan, D.I. Khomskii, and M.V. Mostovoy, Eur. Phys. J. B 12, 217 (1999).

[16] D.I. Golosov, Phys. Rev. Lett. 84, 3974 (2000); J. Appl. Phys. 87, 5804 (2000); ibid. 91, 7508 (2002).

[17] The DEV model lacks the "site-local continuous degeneracy" that is responsible for the phase separation found in Ref.[16].

[18] T. Mizokawa, D.I. Khomskii, and G.A. Sawatzky, Phys. Rev. B 63, 024403 (2000).

[19] The FM coupling in the insulating phase of the manganites is associated with electronic hopping between neighboring $\mathrm{Mn}^{3+}$ and $\mathrm{Mn}^{4+}$ ions but is enhanced by the local polaronic distortions of the $\mathrm{O}$ atoms around some of the $\mathrm{Mn}^{4+}$ ions [18]. The FM Heisenberg coupling $J$ reflects the enhancement of the FM interaction beyond that produced by the uniform hopping of electrons on an undistorted lattice.

[20] T. Asaka, S. Yamada, S. Tsutsumi, C. Tsurata, K. Kimoto, T. Arima, and Y. Matsui, Phys. Rev. Lett. 88, 097201 (2002).

[21] C.J. Halboth and W. Metzner, Phys. Rev. Lett. 85, 5162 (2000).

[22] N. Furukawa, J. Phys. Soc. Japan 64, 2754 (1995); N. Furukawa in Physics of Manganites, edited by T.A. Kaplan and S.D. Mahanti (Plenum, New York, 1999).

[23] R.S. Fishman, (unpublished).

[24] V. Kiryukhin, D. Casa, J.P. Hill, B. Keimer, A. Vigliante, Y. Tomioka, and Y. Tokura, Nature 386, 813 (1997).

[25] S. Mercone, V. Hardy, C. Martin, C. Simon, D. Saurel, and A. Brûlet, Phys. Rev. B 68, $094422(2003)$. 